\title{
THE SPECTRAL POSITION OF NEUMANN DOMAINS ON THE TORUS
}

\author{
RAM BAND ${ }^{1}$, SEBASTIAN K EGGER ${ }^{1}$, ALEXANDER J TAYLOR ${ }^{2}$
}

\begin{abstract}
Neumann domains of Laplacian eigenfunctions form a natural counterpart of nodal domains. The restriction of an eigenfunction to one of its nodal domains is the first Dirichlet eigenfunction of that domain. This simple observation is fundamental in many works on nodal domains. We consider a similar property for Neumann domains. Namely, given a Laplacian eigenfunction $f$ and its Neumann domain $\Omega$, what is the position of $\left.f\right|_{\Omega}$ in the Neumann spectrum of $\Omega$ ?

The current paper treats this spectral position problem on the two-dimensional torus. We fully solve it for separable eigenfunctions on the torus and complement our analytic solution with numerics for random waves on the torus. These results answer questions from $[37,8]$ and arouse new ones.
\end{abstract}

\section{INTRODUCTION}

1.1. Neumann domains. Let $(M, g)$ be a two-dimensional, connected, orientable and closed Riemannian manifold. We denote by $-\Delta$ the (negative) self-adjoint LaplaceBeltrami operator. Its spectrum is purely discrete since $M$ is compact. We order the eigenvalues $\left\{\lambda_{n}\right\}_{n=0}^{\infty}$ increasingly, $0=\lambda_{0}<\lambda_{1} \leq \lambda_{2} \leq \ldots$, and denote a corresponding complete system of orthonormal eigenfunctions by $\left\{f_{n}\right\}_{n=0}^{\infty}$, so that we have

$$
-\Delta f_{n}=\lambda_{n} f_{n} .
$$

Let $f$ be an eigenfunction (we suppress its position, for brevity). We introduce a flow along the gradient vector field, $\nabla f$,

$$
\begin{aligned}
& \varphi: \mathbb{R} \times M \rightarrow M, \\
& \partial_{t} \varphi(t, \boldsymbol{x})=-\left.\nabla f\right|_{\varphi(t, \boldsymbol{x})}, \\
& \varphi(0, \boldsymbol{x})=\boldsymbol{x} .
\end{aligned}
$$

The set of critical points of $f$ is denoted by $\mathscr{C}(f)$; the sets of saddle points and extrema of $f$ are denoted by $\mathscr{S}(f)$ and $\mathscr{X}(f)$; the sets of minima and maxima of $f$ are denoted by $\mathscr{M}_{-}(f)$ and $\mathscr{M}_{+}(f)$, respectively.

For a critical point $\boldsymbol{x} \in \mathscr{C}(f)$, we define its stable and unstable manifolds by

$$
\begin{aligned}
W^{s}(\boldsymbol{x}) & :=\left\{\boldsymbol{y} \in M \quad: \lim _{t \rightarrow \infty} \varphi(t, \boldsymbol{y})=\boldsymbol{x}\right\} \quad \text { and } \\
W^{u}(\boldsymbol{x}) & :=\left\{\boldsymbol{y} \in M: \lim _{t \rightarrow-\infty} \varphi(t, \boldsymbol{y})=\boldsymbol{x}\right\},
\end{aligned}
$$

respectively.

We assume in the following that the eigenfunctions $f$ are Morse functions, i.e., the determinant of the Hessian does not vanish at critical points. We call such an $f$ a Morse eigenfunction. Eigenfunctions are generically Morse, as was shown in [4, 35].

2000 Mathematics Subject Classification. 35Pxx, 57M20.

Key words and phrases. Neumann domains, Neumann lines, nodal domains, Laplacian eigenfunctions, Morse-Smale complexes. 
Definition 1.1. [8] Let $f$ be a Morse function.

(1) Let $\boldsymbol{p} \in \mathscr{M}_{-}(f), \boldsymbol{q} \in \mathscr{M}_{+}(f)$, such that $W^{s}(\boldsymbol{p}) \cap W^{u}(\boldsymbol{q}) \neq \emptyset$. Each of the connected components of $W^{s}(\boldsymbol{p}) \cap W^{u}(\boldsymbol{q})$ is called a Neumann domain of $f$.

(2) The Neumann line set of $f$ is

$$
\mathcal{N}(f):=\overline{\bigcup_{\boldsymbol{r} \in \mathscr{I}(f)} W^{s}(\boldsymbol{r}) \cup W^{u}(\boldsymbol{r})}
$$

Note that the definition above may be applied to any Morse function and not necessarily to eigenfunctions. Nevertheless, in this paper we are interested to study the Neumann domains and Neumann lines of Laplacian eigenfunctions.

It follows from basic Morse theory that Neumann domains are two-dimensional subsets of $M$, whereas the Neumann line set is a union of one dimensional curves on $M$. As an example, see Figure 1.1 which shows an eigenfunction of the flat torus with its partition to Neumann domains.
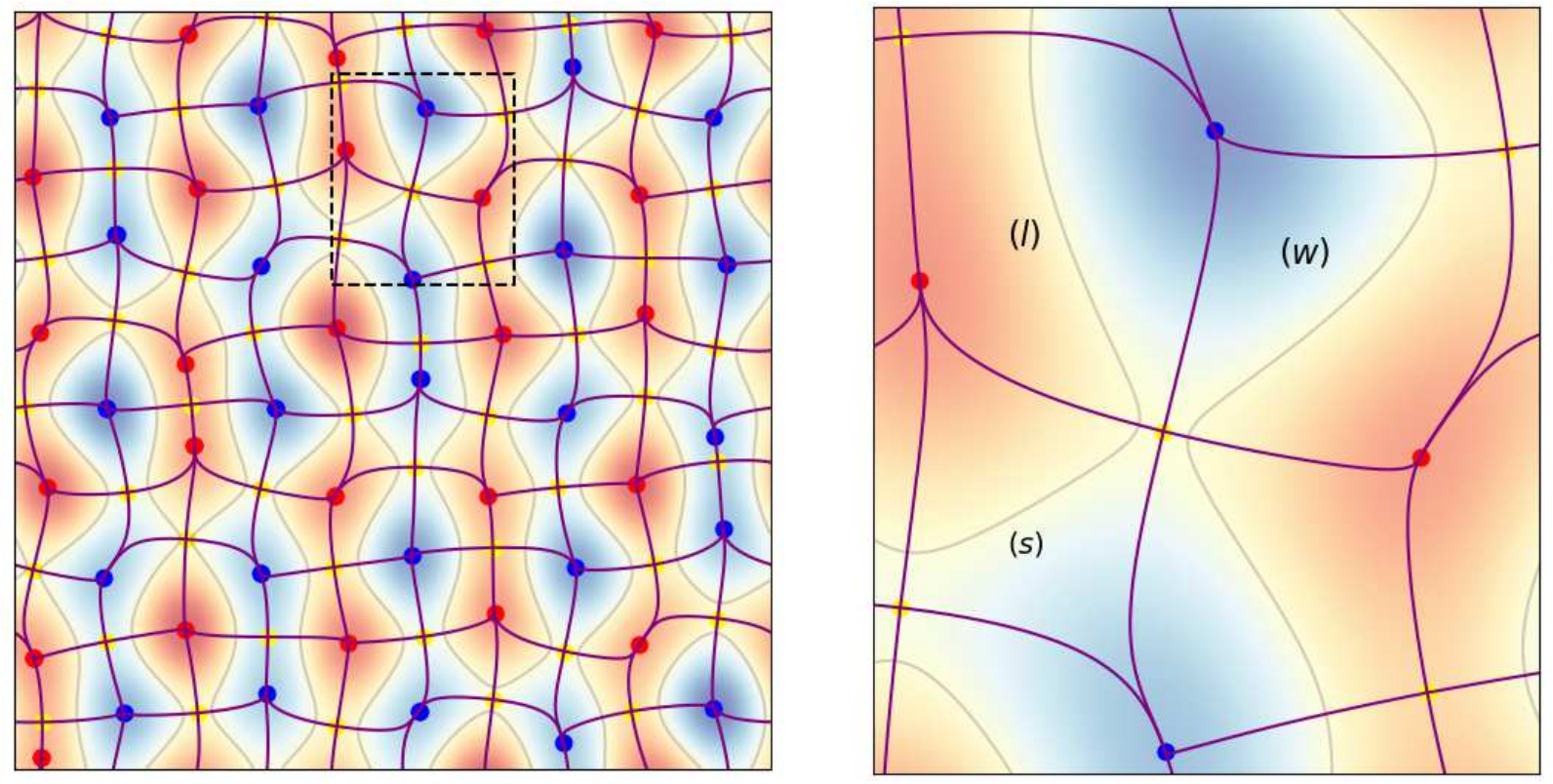

FiguRE 1.1. Left: An eigenfunction corresponding to the eigenvalue $\lambda=25$ of the flat torus whose fundamental domain is $[0,2 \pi] \times[0,2 \pi]$. Red (blue) colors indicate positive (negative) values of the eigenfunction. Red (blue) circles mark maxima (minima) and yellow circles mark saddle points. The nodal set is drawn in grey and the Neumann line set in purple. The Neumann domains are the domains bounded by the Neumann line set.

Right: A magnification of the marked square from the left figure. Three Neumann domains are marked by (s), (l) and (w) (which stand for star, lens and wedge) according to the three distinguished Neumann domain types described in Section 6.3.

1.2. Spectral position . Let $f$ be an eigenfunction corresponding to an eigenvalue $\lambda$ and let $\Omega$ be a Neumann domain of $f$. The boundary, $\partial \Omega$, consists of Neumann lines, which are particular gradient flow lines. As the gradient $\nabla f$ is tangential to the Neumann lines we get that $\left.\partial_{\nu} f\right|_{\partial \Omega}:=\left.\hat{\nu} \cdot \nabla f\right|_{\partial \Omega}=0$, where $\hat{\nu}$ is normal to $\partial \Omega$. As a consequence we have that $\left.f\right|_{\Omega}$ is a Neumann eigenfunction of $\Omega$ and corresponds to the eigenvalue $\lambda$, which is the reason 
behind the name Neumann domains. The proof that $\left.f\right|_{\Omega}$ is a Neumann eigenfunction of a general Neumann domain $\Omega$, goes beyond the classical Sobolev embedding theorems ${ }^{1}$ and appears in [7]. In the current paper we only supply a proof suited for the particular Neumann domains treated here (Proposition 3.4,(2) and Remark 3.5).

Following the discussion above we get that $\lambda$ is a Neumann eigenvalue of $\Omega$. It is natural to ask what is the position of this $\lambda$ in the Neumann spectrum of $\Omega$.

Definition 1.2. Let $f$ be a Morse eigenfunction of an eigenvalue $\lambda$ and let $\Omega$ be a Neumann domain of $f$. We define the spectral position of $\Omega$ as the position of $\lambda$ in the Neumann spectrum of $\Omega$. It is explicitly given by

$$
N_{\Omega}(\lambda):=\left|\left\{\lambda_{n} \in \operatorname{Spec}(\Omega): \lambda_{n}<\lambda\right\}\right|,
$$

where $\operatorname{Spec}(\Omega):=\left\{\lambda_{n}\right\}_{n=0}^{\infty}$ is the pure point part of the Neumann spectrum of $\Omega$, containing multiple appearances of degenerate eigenvalues and including $\lambda_{0}=0$.

Remark 1.3 .

(1) If $\lambda$ is a multiple eigenvalue of $\Omega$, then by this definition the spectral position is the lowest position of $\lambda$ in the spectrum.

(2) The spectral position is positive for any Neumann domain, i.e., $N_{\Omega}(\lambda)>0$. Indeed, $N_{\Omega}(\lambda)=0$ is possible only for $\lambda=0$, but the zero eigenvalue corresponds to the constant eigenfunction which does not have Neumann domains at all.

For comparison, we mention what is the spectral position for nodal domains. Consider a nodal domain $\Xi$ of some eigenfunction $f$ corresponding to an eigenvalue $\lambda$. It is known that $\left.f\right|_{\Xi}$ is the first eigenfunction (aka ground-state) of $\Xi$ with Dirichlet boundary conditions [11]. Namely, $\lambda$ is the lowest eigenvalue in the Dirichlet spectrum of $\Xi$, or if adopting the notation (1.2) for the Dirichlet spectrum we get $N_{\Xi}(\lambda)=0$. This observation is fundamental in many results concerning nodal domains and their counting.

The purpose of this paper is to study the spectral positions of Neumann domains. The general problem is quite involved (comparing to the easy answer for nodal domains, as mentioned above) and in this paper we concentrate on investigating this problem for the two-dimensional flat torus.

A qualitative feeling on the value of $N_{\Omega}(\lambda)$ might be given by [8, Theorem 1.4] (see also [5, Theorem 3.2]). It is shown there that the "topography" of $\left.f\right|_{\Omega}$ cannot be too complex; its domain, $\Omega$, is simply connected; $\left.f\right|_{\Omega}$ has no critical points in the interior of $\Omega$; and its zero set is merely a single simple non-intersecting curve. These observations suggest that $\left.f\right|_{\Omega}$ might not lie too high in the spectrum of $\Omega$. Such a belief appears also in [37], where it is written that possibly, the spectral position of Neumann domains 'often' equals one, just as in the case of nodal domains. Our task is to study the possible values of $N_{\Omega}(\lambda)$ and to find out to what extent $\lambda$ is indeed the first non trivial eigenvalue of $\Omega\left(N_{\Omega}(\lambda)=1\right)$.

1.3. Torus eigenfunctions. We consider the flat torus with fundamental domain $\mathbb{R}^{2} / \mathbb{Z}^{2}$ equipped with the Laplace operator. The eigenvalues are

$$
\lambda_{a, b}:=\frac{\pi^{2}}{4}\left(\frac{1}{a^{2}}+\frac{1}{b^{2}}\right),
$$

where

$$
a:=\frac{1}{4 m_{x}}, b:=\frac{1}{4 m_{y}}, \quad \text { for } m_{x}, m_{y} \in \mathbb{N} .
$$

\footnotetext{
${ }^{1}$ The reason for this is that the boundary of a general Neumann domain might include a cusp and in general we do not have an explicit expression of the cusp.
} 


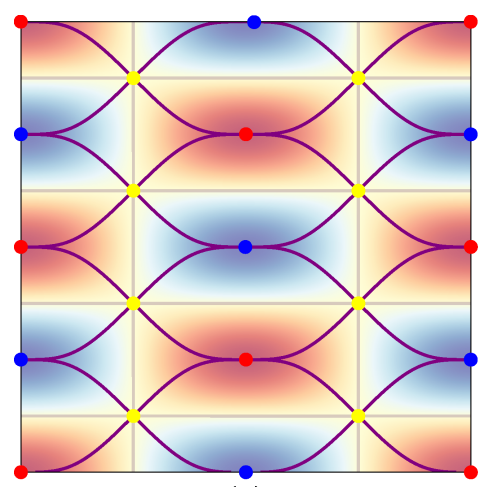

(i)

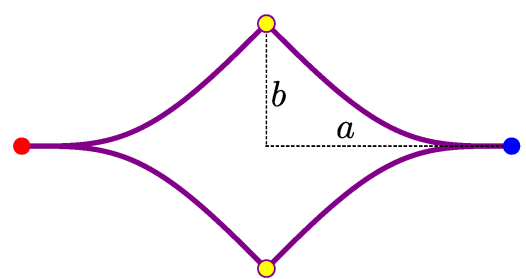

(ii)

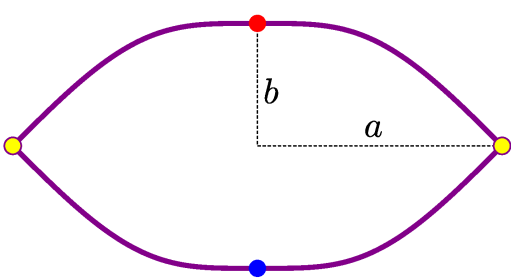

(iii)

Figure 1.2. (i): The torus eigenfunction $f(x, y)=\cos (2 \pi x) \cos (4 \pi y)$ with grey lines indicating its nodal set and purple lines indicating the Neumann line set. (ii) and (iii): the star-like and lens-like Neumann domains of a separable eigenfunction (1.8), with the typical lengths $a, b$ marked as dashed lines. Saddle points are marked by yellow circles and extrema by blue and red circles.

The separable eigenfunctions may be written as

$$
f_{a, b}(x, y)=\cos \left(\frac{\pi}{2 a} x\right) \cos \left(\frac{\pi}{2 b} y\right) .
$$

Each of those eigenfunctions has two types of Neumann domains. Half of them are of a lens shape and congruent to each other and the other half are of a star shape and also congruent (Figure 1.2). We denote those domains by $\Omega_{a, b}^{\text {star }}$ (Figure 1.2,(ii)) and $\Omega_{a, b}^{\text {lens }}$ (Figure 1.2,(iii)), respectively. The size of those Neumann domains is determined by the values of $a, b$ in (1.7). We wish to study the spectral position of those Neumann domains.

First, observe that the symmetry of the problem allows us to consider only the case $b \leq a$. Second, the spectral position of either $\Omega_{a, b}^{\text {star }}$ or $\Omega_{a, b}^{\text {lens }}$ depends only on the ratio $\frac{a}{b}$, as rescaling both $a$ and $b$ by the same factor amounts to an appropriate rescaling of the Neumann domain together with its eigenfunction restriction. We then have the following.

\section{Theorem 1.4.}

(1) The set of spectral positions of the lens-like domains $\left\{N_{\Omega_{a, b}^{\text {lens }}}\left(\lambda_{a, b}\right)\right\}_{a, b}$ is unbounded. In particular, $N_{\Omega_{a, b}^{\text {lens }}}\left(\lambda_{a, b}\right) \rightarrow \infty$ for $\frac{a}{b} \rightarrow \infty$.

(2) There exists $c>1$ such that if $a / b>c$ then the spectral position of the star-like domains equals one, i.e., $N_{\Omega_{a, b}^{\text {star }}}\left(\lambda_{a, b}\right)=1$. In addition $\lambda_{a, b}$ is a simple Neumann eigenvalue of $\Omega_{a, b}^{\text {star }}$.

Remark 1.5. We may provide an estimate for the constant in the second part of the theorem, which is $c \approx 1.1407$. This is done in the course of the theorem's proof (see Remark A.5). That this constant is close to one means that the result of the theorem is quite close to being optimal (since we always assume $a>b$ ). It is interesting to find out whether the result in the theorem actually holds for all $a>b$.

Remark 1.6. In [8, Proposition 1.7] it was proven that $\left\{N_{\Omega_{a, b}^{\text {lens }}}\left(\lambda_{a, b}\right)\right\}_{a, b} \cup\left\{N_{\Omega_{a, b}^{\text {star }}}\left(\lambda_{a, b}\right)\right\}_{a, b}$ is unbounded. The first part of Theorem 1.4 is a refinement of that result, showing that the unboundedness of the spectral position is due to the lens-like Neumann domains. 
The first part of Theorem 1.4 is surprising in the light of the intuition described at the end of Section 1.2. The original expectation was that the spectral positions of Neumann domains would be relatively low, if not even equal to one, as in the case of nodal domains. The theorem above shows that spectral positions may behave completely differently than expected. The second part of the theorem somewhat revives the original intuition about spectral positions. In essence, we show that the unbounded spectral positions of the lenslike domains are compensated by the minimal spectral positions of the other half of the Neumann domains - the star-like ones.

In general, the computation of spectral positions is not an easy task. In particular, it is harder to show that the spectral position is low (rather than high). For example, using test functions in the corresponding quadratic form (aka Rayleigh-Ritz quotient) could only be used to prove lower bounds on spectral positions.

The outline of the paper is as follows. In the next section we bring two proofs of the first part of Theorem 1.4. The proof of the second part of Theorem 1.4 spreads over Sections 3, 4 and 5 . In Section 3 we provide the basic spectral properties of the Neumann eigenvalue problem on $\Omega_{a, b}^{\text {star }}$. Section 4 presents a symmetry reduction of this eigenvalue problem. Section 5 then complements the proof by solving some required eigenvalue comparison problems. In Section 6 we go beyond separable eigenfunctions and combine a useful geometric parameter with numerical methods to study the generic behavior of spectral positions. Some technical computations needed for the proofs are deferred to Appendix A.

\section{Two proofs of Theorem 1.4, PART (1)}

First proof of Theorem 1.4, (1). Assume by contradiction that there exists a constant $M$ such that $\forall a, b \quad \lambda_{a, b} \leq \lambda_{M}\left(\Omega_{a, b}^{\text {lens }}\right)$. By [19] we have $\lambda_{M}\left(\Omega_{a, b}^{\text {lens }}\right)\left|\Omega_{a, b}^{\text {lens }}\right| \leq 8 \pi M$, where $\left|\Omega_{a, b}^{\text {lens }}\right|$ denotes the area of $\Omega_{a, b}^{\text {lens }}$. Combining this with the contradiction assumption we get

$$
\forall a, b \quad \lambda_{a, b}\left|\Omega_{a, b}^{\text {lens }}\right| \leq 8 \pi M .
$$

In addition,

$$
\begin{aligned}
\forall a, b \quad \lambda_{a, b}\left|\Omega_{a, b}^{\text {lens }}\right| & =\frac{\pi^{2}}{4}\left(\frac{1}{a^{2}}+\frac{1}{b^{2}}\right)\left|\Omega_{a, b}^{\text {lens }}\right| \\
& =\pi^{2}\left(\frac{a}{b}+\frac{b}{a}\right)\left(\frac{1}{4 a b}\left|\Omega_{a, b}^{\text {lens }}\right|\right) \\
& =\pi^{2}\left(\frac{a}{b}+\frac{b}{a}\right)\left(\frac{1}{4 a b}\left(4 a b-\left|\Omega_{a, b}^{\text {star }}\right|\right)\right) \\
& >\pi^{2}\left(\frac{a}{b}+\frac{b}{a}\right)-\frac{\pi^{2}}{4} \frac{2}{\pi}\left(j_{0}\right)^{2}
\end{aligned}
$$

where we used that $\left|\Omega_{a, b}^{\text {lens }}\right|+\left|\Omega_{a, b}^{\text {star }}\right|=4 a b$ (since the union of a quarter of $\Omega_{a, b}^{\text {lens }}$ and a quarter of $\Omega_{a, b}^{\text {star }}$ gives a rectangle $a \times b$ ) and the last line is a consequence of Lemma A.5.

Taking the limit $\frac{a}{b} \rightarrow \infty$ in (2.2) we get $\lambda_{a, b}\left|\Omega_{a, b}^{\text {lens }}\right| \rightarrow \infty$, which contradicts (2.1). Hence $\left\{N_{\Omega_{a, b}^{\text {lens }}}\left(\lambda_{a, b}\right)\right\}_{a, b}$ is unbounded. 
Second proof of Theorem 1.4, (1). The lens domain $\Omega_{a, b}^{\text {lens }}$ is bounded within a rectangle of width $2 a$ and height $2 b$ (see Figure 1.2,(iii)). When taking the limit $b \rightarrow 0$ the lens domain shrinks into a one edge graph of length $2 a$. Applying results from [21, 20, 33] (for example, we may use [21, Theorem 3.1] with $\alpha=1$ ) we get the eigenvalue convergence

$$
\lim _{b \rightarrow 0} \lambda_{n}\left(\Omega_{a, b}^{\text {lens }}\right)=\left(\frac{\pi}{2 a} n\right)^{2} .
$$

Comparing this with the eigenvalue of the separable eigenfunction

$$
\lambda_{a, b}=\frac{\pi^{2}}{4}\left(\frac{1}{a^{2}}+\frac{1}{b^{2}}\right),
$$

we see that when fixing the value of $a$ and letting $b \rightarrow 0$ the spectral position of $\lambda_{a, b}$ in the spectrum of $\Omega_{a, b}^{\text {lens }}$ is indeed unbounded.

\section{BASIC SPECTRAL ANALYSIS ON $\Omega_{a, b}^{\mathrm{star}}$}

In this section we state and prove some basic spectral properties of the Neumann domain $\Omega_{a, b}^{\text {star }}$ which are needed for the proof of Theorem 1.4,(2).

The domain $\Omega_{a, b}^{\text {star }}$ is given by

$$
\Omega_{a, b}^{\mathrm{star}}=\left\{(x, y):-a<x<a,-\gamma_{a, b}(x)<y<\gamma_{a, b}(x)\right\}
$$

where

$$
\gamma_{a, b}(x):=\frac{2 b}{\pi} \arcsin \left(\left[\cos \left(\frac{\pi}{2 a} x\right)\right]^{\left(\frac{a}{b}\right)^{2}}\right) .
$$

See Lemma A.1 where the boundary curve $\gamma_{a, b}$ is explicitly calculated.

In order to analyze the Neumann spectrum of $\Omega_{a, b}^{\text {star }}$ we need a suitable description of the operator and the relevant quadratic form. The domain $\Omega_{a, b}^{\text {star }}$ possesses a cusp (at $x= \pm a$, $y=0$ ), which prohibits the standard application of the Gauss-Green identity (integration by parts), cf. [2] and prevents a direct characterization of the Neumann Laplacian in terms of Neumann boundary conditions. Instead, we use the approach which describes a semibounded self-adjoint operator by its uniquely associated quadratic form.

We start by introducing a sesquilinear form on $\Omega_{a, b}^{\text {star }}$ which then generates in a canonical way ([31, Theorem VIII.15]) the operator $\Delta_{a, b}$, which is the self-adjoint Neumann Laplacian on $\Omega_{a, b}^{\text {star }}$. The form and its domain are

$$
\begin{aligned}
q[\phi, \psi] & :=\int_{\Omega_{a, b}^{\mathrm{star}}}\langle\nabla \psi(x), \nabla \phi(x)\rangle_{\mathbb{C}^{2}} \mathrm{~d} x, \\
\operatorname{Dom}(q) & :=W^{1,2}\left(\Omega_{a, b}^{\mathrm{star}}\right),
\end{aligned}
$$

where $W^{1,2}\left(\Omega_{a, b}^{\text {star }}\right)$ denotes the corresponding Sobolev space on $\Omega_{a, b}^{\text {star }}$. Indeed, $q[\cdot, \cdot]$ yields an appropriate and well-defined Laplacian as the following proposition shows.

\section{Proposition 3.1.}

(1) $(q, \operatorname{Dom}(q))$ defines a unique self-adjoint operator $\Delta_{a, b}$,

(2) $\Delta_{a, b}$ has purely discrete spectrum.

Proof. The standard approach [31, Theorem VIII.15] to verify part (1) is to realize that the form is non-negative and to show that the form domain, $\operatorname{Dom}(q)$ is complete under the form norm $\|\cdot\|_{q}:=\left(q[\cdot, \cdot]+\|\cdot\|_{L^{2}\left(\Omega_{a, b}^{\text {star }}\right)}\right)^{\frac{1}{2}}$. Indeed, the latter coincides with the standard 
Sobolev space norm on $W^{1,2}\left(\Omega_{a, b}^{\mathrm{star}}\right)$, i.e. $\|\varphi\|_{W^{1,2}\left(\Omega_{a, b}^{\mathrm{star}}\right)}=\|\varphi\|_{q}$. Therefore, the completeness of $W^{1,2}\left(\Omega_{a, b}^{\text {star }}\right)$ implies the completeness of the form domain.

To prove part (2) we start by noting that according to Lemma A.3 the boundary $\partial \Omega_{a, b}^{\text {star }}$ is of class $C$. This is equivalent to $\Omega_{a, b}^{\text {star }}$ having the segment property [12, Theorem V.4.4]

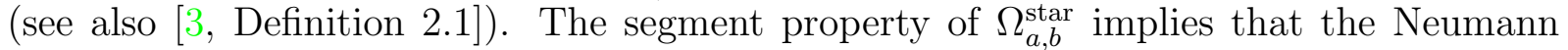
Laplacian, $\Delta_{a, b}$ has a compact resolvent [32, Corollary 1 of Theorem XIII.75] and this is equivalent to the discreteness of the spectrum [32, Theorem XIII.64].

Remark 3.2. Note that the operator $\Delta_{a, b}$ acts as the standard (negative) weak Laplacian, as can be shown by using $C_{0}^{\infty}\left(\Omega_{a, b}^{\text {star }}\right)$ functions to cut away the cusp and integrating by parts.

Remark 3.3. The statements of Proposition 3.1 analogously hold for the domain $\Omega_{a, b}^{\text {lens }}$ as well. Indeed, the boundary $\partial \Omega_{a, b}^{\text {lens }}$ possesses no cusp and standard Lipschitz domain arguments can be applied to show such statements.

We now investigate the operator domain $\operatorname{Dom}\left(\Delta_{a, b}\right)$ in more detail and show that the restrictions $\left.f_{a, b}\right|_{\Omega_{a, b}^{\text {star }}}$ belong to $\operatorname{Dom}\left(\Delta_{a, b}\right)$, which justifies the definition of the spectral position for $\Omega_{a, b}^{\mathrm{star}}$.

\section{Proposition 3.4.}

(1) The operator domain $\operatorname{Dom}\left(\Delta_{a, b}\right)$ satisfies

$$
\operatorname{Dom}\left(\Delta_{a, b}\right) \subset\left\{f \in W^{2,2}\left(\Omega_{a, b}^{\mathrm{star}}\right):\left.\partial_{\nu} f\right|_{\partial \Omega_{a, b}^{\mathrm{star}}} \equiv 0\right\}
$$

where $\left.\partial_{\nu} f\right|_{\partial \Omega_{a, b}^{\mathrm{star}}}$ is the normal derivative.

(2) Every separable eigenfunction $f_{a, b}$ satisfies $\left.f_{a, b}\right|_{\Omega_{a, b}^{\text {star }}} \in \operatorname{Dom}\left(\Delta_{a, b}\right)$.

Hence, $\left.f_{a, b}\right|_{\Omega_{a, b}^{\text {star }}}$ is an eigenfunction of $\Delta_{a, b}$.

Proof. Part (1). We start by showing $\operatorname{Dom}\left(\Delta_{a, b}\right) \subset W^{2,2}\left(\Omega_{a, b}^{\text {star }}\right)$. Let $f \in \operatorname{Dom}\left(\Delta_{a, b}\right)$ and denote $g:=\Delta_{a, b} f$. We may use [24, Proposition 8.3.2] to conclude that there is a unique $W^{1,2}\left(\Omega_{a, b}^{\text {star }}\right)$ solution $\psi$ (up to an additive constant function) for the equation $g=\Delta_{a, b}(\psi)$. Explicitly, for the application of [24, Proposition 8.3.2] we take $q=q^{\prime}=1, l=1$, $a_{\alpha, \beta}(x) \equiv \delta_{\alpha, \beta}$ (Kronecker delta function) and verify that the assumption on the boundary near the outer cusp (peak) is satisfied since $\gamma_{a, b}(a)=0$ and $\lim _{x \rightarrow a} \gamma_{a, b}^{\prime}(x)=0$ (see (3.1)). Next, we use an elliptic regularity result of [18, Remark 3.3.3] to conclude that the equation $g=\Delta_{a, b}(\psi)$ has a unique $W^{2,2}\left(\Omega_{a, b}^{\text {star }}\right)$ solution (up to an additive constant function). From $W^{2,2}\left(\Omega_{a, b}^{\text {star }}\right) \subset W^{1,2}\left(\Omega_{a, b}^{\text {star }}\right)$ and the uniqueness of the $W^{1,2}\left(\Omega_{a, b}^{\text {star }}\right)$ solution we conclude that those solutions are the same and since $f \in \operatorname{Dom}\left(\Delta_{a, b}\right) \subset W^{1,2}\left(\Omega_{a, b}^{\text {star }}\right)$ we get that this unique solution is $\psi=f$ and $f \in W^{2,2}\left(\Omega_{a, b}^{\text {star }}\right)$. To apply the regularity result of [18, Remark 3.3.3] we need to verify that the condition imposed there on the boundary is satisfied. In terms of the notations of [18, Remark 3.3.3] we have $-\phi_{1}(x)=\phi_{2}(x)=\gamma_{a, b}(a-x)$ and the condition on these functions may be verified with the aid of (A.4).

All is left to show is that $\left.\partial_{\nu} f\right|_{\partial \Omega_{a, b}^{\text {star }}} \equiv 0$. This is done by employing standard localization techniques, as follows. Let $z \in \partial \Omega_{a, b}^{\text {star }}$ not identical to a cusp point (i.e., $z \notin\{(0, a),(0,-a)\})$. Then there exists a neighborhood of $z$, say a disc $B \subset \mathbb{R}^{2}$ which does not contain any of the cusp points of $\Omega_{a, b}^{\text {star }}$. Choose a localizing non-negative $C^{\infty}$ function such that $\operatorname{supp} \tilde{\eta}=\bar{B}$. Denote $\eta:=\left.\tilde{\eta}\right|_{\Omega_{a, b}^{\text {star }}}$. 
For all $f \in \operatorname{Dom}\left(\Delta_{a, b}\right)$ and $\varphi \in W^{1,2}\left(\Omega_{a, b}^{\text {star }}\right)$ we have

$$
\begin{aligned}
\left\langle\Delta_{a, b} f, \eta \varphi\right\rangle_{L^{2}\left(\Omega_{a, b}^{\mathrm{star}}\right)} & =q[f, \eta \varphi] \\
& =\int_{\Omega_{a, b}^{\mathrm{star}}}\langle\nabla f, \nabla(\eta \varphi)\rangle_{\mathbb{C}^{2}} \mathrm{~d} x \\
& =\int_{\operatorname{supp} \eta}\langle\nabla f, \nabla(\eta \varphi)\rangle_{\mathbb{C}^{2}} \mathrm{~d} x \\
& =-\int_{\operatorname{supp} \eta} \overline{\operatorname{div} \nabla f} \eta \varphi \mathrm{d} x+\int_{\partial\left(B \cap \Omega_{a, b}^{\mathrm{star}}\right)}\left(\partial_{\nu} f\right)(\eta \varphi) \mathrm{d} \sigma \\
& =-\int_{\Omega_{a, b}^{\mathrm{star}}} \overline{\operatorname{div} \nabla f} \eta \varphi \mathrm{d} x+\int_{\partial\left(B \cap \Omega_{a, b}^{\mathrm{star}}\right)}\left(\partial_{\nu} f\right)(\eta \varphi) \mathrm{d} \sigma \\
& =\left\langle\Delta_{a, b} f, \eta \varphi\right\rangle_{L^{2}\left(\Omega_{a, b}^{\mathrm{star}}\right)}+\int_{\partial\left(B \cap \Omega_{a, b}^{\mathrm{star}}\right)}\left(\partial_{\nu} f\right)(\eta \varphi) \mathrm{d} \sigma,
\end{aligned}
$$

where we stick to the $\operatorname{div} \nabla$ notation and do not use the standard Laplacian notation in order to distinguish this from $\Delta_{a, b}$.

From (3.4) we get that the boundary integral vanishes. By calculus of variations we conclude that $\left.\partial_{\nu} f\right|_{\partial\left(B \cap \Omega_{a, b}^{\text {star }}\right)}=0$ since $\left\{\left.\eta \varphi\right|_{\partial\left(B \cap \Omega_{a, b}^{\text {star }}\right)}: \varphi \in W^{1,2}\left(B \cap \Omega_{a, b}^{\text {star }}\right)\right\}$ is dense in $L^{2}\left(\partial\left(B \cap \Omega_{a, b}^{\text {star }}\right)\right)$. In particular $\partial_{\nu} f(z)=0$, as required.

To prove part (2) we first truncate the domain $\Omega_{a, b}^{\text {star }}$ to remove the cusps. To this end we define the following family of auxiliary domain

$$
\Omega_{\delta}:=\left\{(x, y) \in \Omega_{a, b}^{\mathrm{star}}:|x|<a-\delta\right\},
$$

and notice that for every $\delta>0, \Omega_{\delta}$ is a Lipschitz domain. Let $\psi \in C^{\infty}\left(\overline{\Omega_{a, b}^{\text {star }}}\right) \subset W^{1,2}\left(\Omega_{a, b}^{\mathrm{star}}\right)$ be an arbitrary test function. Then

$$
\begin{aligned}
q\left[f_{a, b}, \psi\right] & =\lim _{\delta \rightarrow 0} \int_{\Omega_{\delta}}\left\langle\nabla f_{a, b}, \nabla \psi\right\rangle_{\mathbb{C}^{2}} \mathrm{~d} x \\
& =\lim _{\delta \rightarrow 0}\left[-\int_{\Omega_{\delta}}\left(\operatorname{div} \nabla f_{a, b}\right) \psi \mathrm{d} x+\int_{\partial \Omega_{\delta} \backslash \partial \Omega}\left(\partial_{\nu} f_{a, b}\right) \psi \mathrm{d} \sigma\right] \\
& =-\int_{\Omega_{a, b}^{\text {star }}}\left(\operatorname{div} \nabla f_{a, b}\right) \psi \mathrm{d} x,
\end{aligned}
$$

where moving to the last line we have used that both $f_{a, b}$ and $\operatorname{div} \nabla f_{a, b}$ are bounded, so that the first integral in the limit converges to an integral over $\Omega_{a, b}^{\text {star }}$ and the boundary integral vanishes in the limit.

As is mentioned in the proof of Proposition 3.1,(2) the domain $\Omega_{a, b}^{\text {star }}$ has the segment property. This implies that $C^{\infty}\left(\overline{\Omega_{a, b}^{\mathrm{star}}}\right)$ is dense in $W^{1,2}\left(\Omega_{a, b}^{\mathrm{star}}\right)$ [3, Theorem 2.1]. Hence we get from (3.5) that

$$
\forall \psi \in W^{1,2}\left(\Omega_{a, b}^{\mathrm{star}}\right), \quad q\left[f_{a, b}, \psi\right]=-\int_{\Omega_{a, b}^{\mathrm{star}}}\left(\operatorname{div} \nabla f_{a, b}\right) \psi \mathrm{d} x .
$$

Since $\operatorname{Dom}(q)=W^{1,2}\left(\Omega_{a, b}^{\text {star }}\right)$ we get from (3.6) that $\left.f_{a, b}\right|_{\Omega_{a, b}^{\mathrm{star}}} \in \operatorname{Dom}\left(\Delta_{a, b}\right)$ and $\Delta_{a, b} f_{a, b}=$ $-\operatorname{div} \nabla f_{a, b}=\lambda_{a, b} f_{a, b}$. 
Remark 3.5. The Neumann Laplacian on the lens domain, $\Omega_{a, b}^{\text {lens }}$ satisfies similar properties, as can be shown with analogous arguments. In fact, using the Lipschitz property of the boundary of $\Omega_{a, b}^{\text {lens }}$, one can employ standard arguments to show equality in (3.3), explicitly characterizing the operator domain.

\section{Symmetry BASEd Analysis towards Proof of Theorem 1.4,(2)}

In the previous section we have shown that there exists a natural self-adjoint Neumann Laplacian on $\Omega_{a, b}^{\text {star }}$, which we denote by $\Delta_{a, b}$ and that the spectrum of $\Delta_{a, b}$ is purely discrete (Proposition 3.1). Next, we describe a spectral decomposition of $\Delta_{a, b}$ based on the symmetry of $\Omega_{a, b}^{\text {star }}$, which would eventually lead to the proof of Theorem $1.4,(2)$.

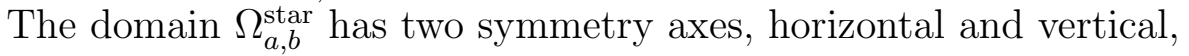

$$
h:=\{(x, y): y=0\} \quad \text { and } \quad v:=\{(x, y): x=0\}
$$

and those dissect $\Omega_{a, b}^{\text {star }}$ into four quarters (see Figure 4.1).

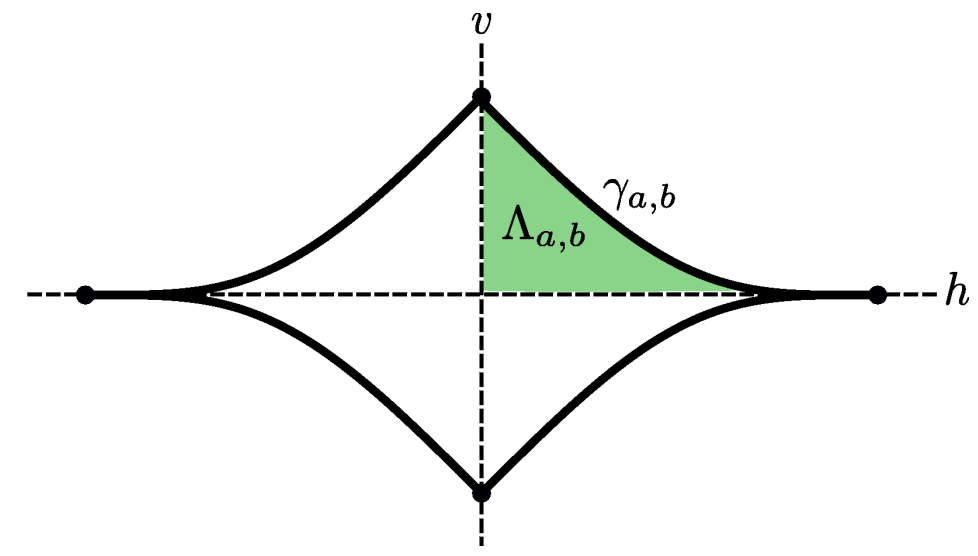

FiguRE 4.1. The star-like domain, $\Omega_{a, b}^{\text {star }}$ with its two symmetry axes, $h, v$. The upper-right quarter, $\Lambda_{a, b}$, is shaded.

We denote by $\Lambda_{a, b}$ the upper-right quarter,

$$
\Lambda_{a, b}=\left\{(x, y): 0<x<a, 0<y<\gamma_{a, b}(x)\right\},
$$

and note that $\Lambda_{a, b}$ is bounded by $\gamma_{a, b}, h$ and $v$, i.e.,

$$
\partial \Lambda_{a, b} \subset h \cup v \cup \gamma_{a, b} .
$$

Next, we introduce four Laplace-Beltrami operators on $\Lambda_{a, b}$, which differ only in their boundary conditions. We denote those Laplacians by $\Delta_{a, b}^{\emptyset}, \Delta_{a, b}^{h}, \Delta_{a, b}^{v}$ and $\Delta_{a, b}^{h \cup v}$, and also use the notation $\Delta_{a, b}^{D}$ when we want to refer to either of them $(D \in\{\emptyset, h, v, h \cup v\})$. The superscripts of those Laplacians indicate which part of the boundary $\partial \Lambda_{a, b}$ serves as the Dirichlet boundary, whereas the rest of the boundary is taken to be Neumann (see Figure 4.2).

Using Proposition 2 in [27] or Property II,(3) in [6] we obtain the following spectral decomposition $^{2}$

$$
\operatorname{Spec}\left(\Delta_{a, b}\right)=\operatorname{Spec}\left(\Delta_{a, b}^{\emptyset}\right) \cup \operatorname{Spec}\left(\Delta_{a, b}^{h}\right) \cup \operatorname{Spec}\left(\Delta_{a, b}^{v}\right) \cup \operatorname{Spec}\left(\Delta_{a, b}^{h \cup v}\right) .
$$

The equality above holds also when taking into account the multiplicities of eigenvalues on both sides.

\footnotetext{
${ }^{2}$ To apply the theory in $[27,6]$ for our case, we take the group to be $C_{2} \times C_{2}$ (the direct product of two copies of the cyclic group, $C_{2}$ ) with its regular representation.
} 

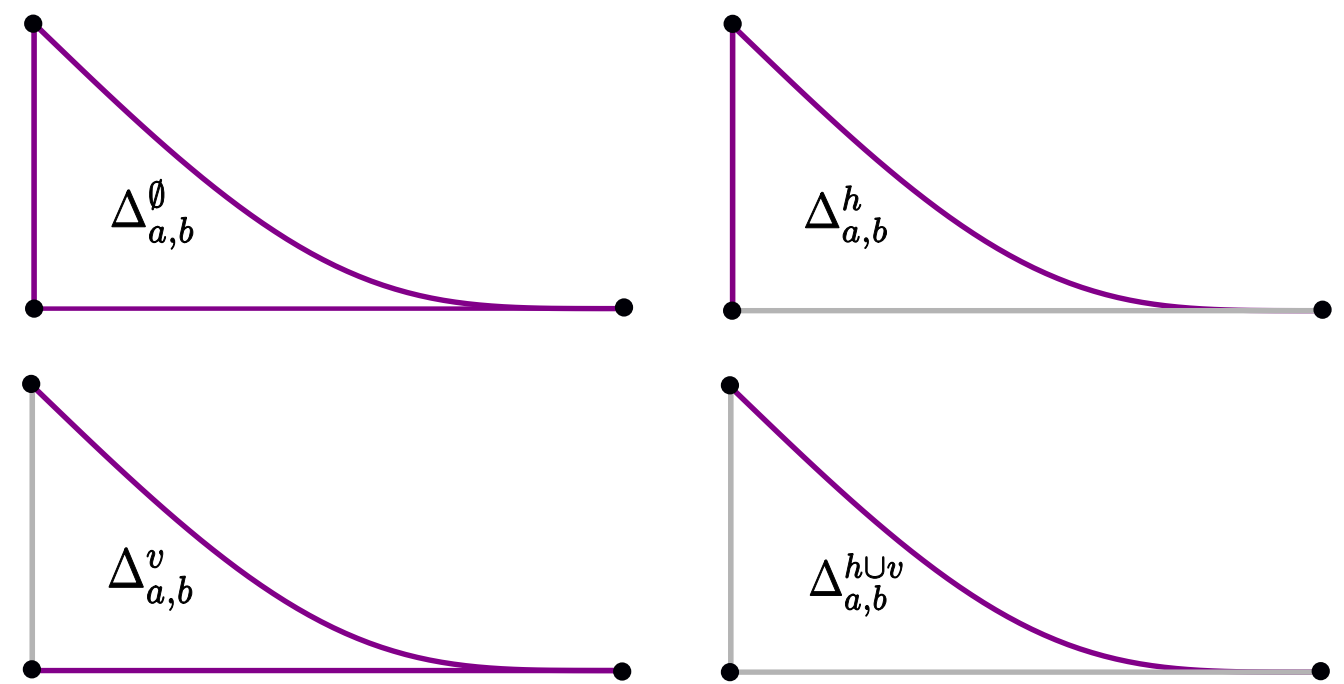

FiguRE 4.2. The four Laplacians $\Delta_{a, b}^{\emptyset}, \Delta_{a, b}^{h}, \Delta_{a, b}^{v}$ and $\Delta_{a, b}^{h \cup v}$, all defined on $\Lambda_{a, b}$ and differ only in boundary conditions. Purple color indicates Neumann conditions and gray stands for Dirichlet.

The spectral decomposition (4.4) may be also understood on an intuitive level, as follows. Since the domain $\Omega_{a, b}^{\text {star }}$ is symmetric with respect to reflection along both $h$ and $v$ we get that that the Neumann Laplacian, $\Delta_{a, b}$ commutes with each of those symmetries. As a result, $\Delta_{a, b}$ posseses a complete set of eigenfunctions which respects this symmetry. Namely, each eigenfunction in this basis is either symmetric or anti-symmetric with respect to $h$ and either symmetric or anti-symmetric with respect to $v$. Each eigenfunction therefore belongs to one of four symmetry classes and its corresponding eigenvalue belongs to either of the spectra $\operatorname{Spec}\left(\Delta_{a, b}^{\emptyset}\right), \operatorname{Spec}\left(\Delta_{a, b}^{h}\right), \operatorname{Spec}\left(\Delta_{a, b}^{v}\right), \operatorname{Spec}\left(\Delta_{a, b}^{h \cup v}\right)$.

Now, Theorem 1.4,(2) follows when combining the spectral decomposition (4.4) together with the following two propositions (the propositions are proven in the next section).

Proposition 4.1.

$$
\lambda_{1}\left(\Delta_{a, b}\right) \notin \operatorname{Spec}\left(\Delta_{a, b}^{\emptyset}\right) \cup \operatorname{Spec}\left(\Delta_{a, b}^{h \cup v}\right)
$$

Proposition 4.2. There exists $c>1$ such that if $a / b>c$ then

$$
\lambda_{1}\left(\Delta_{a, b}^{v}\right)<\lambda_{1}\left(\Delta_{a, b}^{h}\right) .
$$

Proof of Theorem 1.4,(2). From the spectral decomposition (4.4) and Proposition 4.1 we get that either $\lambda_{1}\left(\Delta_{a, b}\right)=\lambda_{1}\left(\Delta_{a, b}^{h}\right)$ or $\lambda_{1}\left(\Delta_{a, b}\right)=\lambda_{1}\left(\Delta_{a, b}^{v}\right)$. Then, by (4.5) we deduce that actually $\lambda_{1}\left(\Delta_{a, b}\right)=\lambda_{1}\left(\Delta_{a, b}^{v}\right)$ for $a / b>c$.

Now, assume $a / b>c$ and consider the eigenfunction $f_{a, b}$ corresponding to $\lambda_{a, b}$ (see (1.8)). The restriction $\left.f_{a, b}\right|_{\Omega_{a, b}^{\text {star }}}$ is symmetric with respect to $h$ and anti-symetric with respect to $v$ and hence $\lambda_{a, b} \in \operatorname{Spec}\left(\Delta_{a, b}^{v}\right)$. Furthermore, the restriction to the quarter star, $\left.f_{a, b}\right|_{\Lambda_{a, b}}$ has a single nodal domain so it is the first eigenfunction of $\Delta_{a, b}^{v}$ (this follows from Courant's bound [11] together with orthogonality of eigenfunctions), i.e., $\lambda_{a, b}=\lambda_{1}\left(\Delta_{a, b}^{v}\right)$. Combining this with $\lambda_{1}\left(\Delta_{a, b}\right)=\lambda_{1}\left(\Delta_{a, b}^{v}\right)$ which we obtained above, we get $\lambda_{a, b}=\lambda_{1}\left(\Delta_{a, b}\right)$, so that $N_{\Omega_{a, b}^{\text {star }}}\left(\lambda_{a, b}\right)=1$, as required.

Finally, the simplicity of $\lambda_{a, b}$ as an eigenvalue of $\Delta_{a, b}$ also follows from the arguments above. We got that $\lambda_{1}\left(\Delta_{a, b}\right)=\lambda_{1}\left(\Delta_{a, b}^{v}\right)$ and also that $\lambda_{1}\left(\Delta_{a, b}\right) \notin \operatorname{Spec}\left(\Delta_{a, b}^{\emptyset}\right) \cup \operatorname{Spec}\left(\Delta_{a, b}^{h}\right) \cup$ 
$\operatorname{Spec}\left(\Delta_{a, b}^{h \cup v}\right)$. By the spectral decomposition (4.4), this means that $\lambda_{1}\left(\Delta_{a, b}\right)$ may be a multiple eigenvalue only if $\lambda_{1}\left(\Delta_{a, b}^{v}\right)$ itself is a multiple eigenvalue of $\Delta_{a, b}^{v}$. But $\lambda_{1}\left(\Delta_{a, b}^{v}\right)$ is the lowest eigenvalue of $\Delta_{a, b}^{v}$ and hence must be simple.

\section{Proofs of Propositions 4.1 And 4.2}

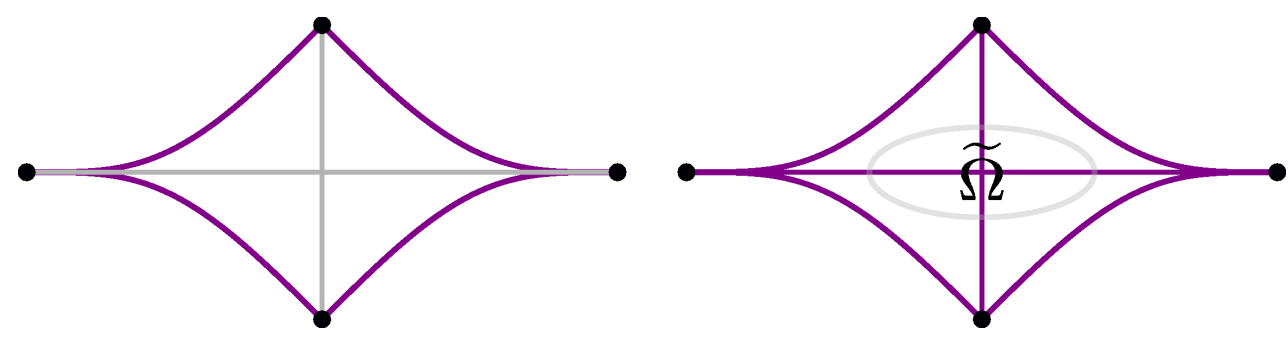

FIGURE 5.1. Depicting properties of particular eigenfunctions of $\Delta_{a, b}$. Grey curves indicate the nodal set of the eigenfunciton and purplue curves mark vanishing of the normal derivative.

Left: An eigenfunction which is anti-symmetric both with respect to $h$ and $\bar{v}$ (so that the corresponding eigenvalue satisfies $\left.\lambda \in \operatorname{Spec}\left(\Delta_{a, b}^{h \cup v}\right)\right)$.

Right: An eigenfunction which is symmetric both with respect to $h$ and $v$ (so that the corresponding eigenvalue satisfies $\lambda \in \operatorname{Spec}\left(\Delta_{a, b}^{\emptyset}\right)$ ).

Proof of Proposition 4.1. First, assume by contradiction that $\lambda_{1}\left(\Delta_{a, b}\right) \in \operatorname{Spec}\left(\Delta_{a, b}^{h \cup v}\right)$. This means that there exists an eigenfunction $f$ corresponding to $\lambda_{1}\left(\Delta_{a, b}\right)$, such that $f$ is anti-symmetric both with respect to the $h$ axis and the $v$ axis. In particular, the nodal set of $f$ contains both $h$ and $v$ and therefore $f$ has at least four nodal domains (see Figure 5.1(Left)). This brings to a contradiction, since by Courant's bound [11], each eigenfunction which corresponds to $\lambda_{1}\left(\Delta_{a, b}\right)$ has at most two nodal domains (note that $\lambda_{0}\left(\Delta_{a, b}\right)=0$, so that $\lambda_{1}\left(\Delta_{a, b}\right)$ is the second lowest eigenvalue).

Next, assume by contradiction that $\lambda_{1}\left(\Delta_{a, b}\right) \in \operatorname{Spec}\left(\Delta_{a, b}^{\emptyset}\right)$. This means that there exists an eigenfunction $f$ corresponding to $\lambda_{1}\left(\Delta_{a, b}\right)$, such that $f$ is symmetric both with respect to the $h$ axis and the $v$ axis. As above, $f$ must have exactly two nodal domains (it cannot have a single nodal domain, as $f$ must be orthogonal to the constant eigenfunction). The only possibility for $f$ to be symmetric as above and contain two nodal domains is if $f$ has a single closed nodal line which is symmetric with respect both to the $h$ axis and the $v$ axis (see Figure 5.1(Right)). This brings to a contradiction, as the first non-trivial Neumann eigenfunction cannot have a closed nodal line [29] (see also [28]). There is a simple argument for that, which we bring here for completeness. We denote the interior nodal domain of $f$ (the one which does not touch the boundary) by $\widetilde{\Omega}$. In particular we get that $\left.f\right|_{\widetilde{\Omega}}$ is a Dirichlet eigenfunction of $\widetilde{\Omega}$ whose eigenvalue is $\lambda_{1}\left(\Delta_{a, b}\right)$. Furthermore, $\left.f\right|_{\widetilde{\Omega}}$ has a single nodal domain and hence it is the first Dirichlet eigenfunction of $\widetilde{\Omega}$, i.e., $\lambda_{1}\left(\Delta_{a, b}\right)=\lambda_{1}^{(D)}(\widetilde{\Omega})$ (this follows from Courant's bound [11] together with eigenfunction orthogonality). If we now denote by $\lambda_{1}^{(D)}\left(\Omega_{a, b}^{\text {star }}\right)$ the first Dirichlet eigenvalue of $\Omega_{a, b}^{\text {star }}$, we get

$$
\lambda_{1}^{(D)}\left(\Omega_{a, b}^{\mathrm{star}}\right) \leq \lambda_{1}^{(D)}(\widetilde{\Omega})=\lambda_{1}\left(\Delta_{a, b}\right)<\lambda_{1}^{(D)}\left(\Omega_{a, b}^{\mathrm{star}}\right),
$$

where the left inequality above follows from the monotonicity of Dirichlet eigenvalues and the right inequality appears already in [30] (see also [28] and note that it is actually part of a more general interlacing property of Dirichlet and Neumann eigenvalues [15, 16]). 
Overall, (5.1) is a contradiction, which means that $f$ cannot be symmetric with respect to both the $v$ and the $h$ axes and $\lambda_{1}\left(\Delta_{a, b}\right) \notin \operatorname{Spec}\left(\Delta_{a, b}^{\emptyset}\right)$.

Next, to prove Proposition 4.2 we need to compare the first eigenvalues of the operators $\Delta_{a, b}^{v}$ and $\Delta_{a, b}^{h}$. We do so by using a sector as an auxiliary domain and proving that its first eigenvalue lies in between $\lambda_{1}\left(\Delta_{a, b}^{v}\right)$ and $\lambda_{1}\left(\Delta_{a, b}^{h}\right)$. This is done in Lemmata 5.1 and 5.2 and for this purpose we denote

$$
S_{R}:=\{(r \cos (\phi), r \sin (\phi)): \quad 0<r<R,|\phi|<\pi / 8\} .
$$

In particular, we choose $R$ to be such that the sector area equals the area of $\Omega_{a, b}^{\mathrm{star}}$,i.e., $\left|S_{R}\right|=\frac{\pi}{8} R^{2}=\left|\Lambda_{a, b}\right|$. We consider the Laplacian on $S_{R}$ with

Neumann boundary conditions on $\{(r \cos (\phi), r \sin (\phi)): 0<r<R,|\phi|=\pi / 8\}$

and Dirichlet boundary conditions on $\{(R \cos (\phi), R \sin (\phi)):|\phi|<\pi / 8\}$.

We denote the first eigenvalue of this Laplacian on the sector by $\lambda_{1}\left(S_{R}\right)$.

Lemma 5.1. We have

$$
\lambda_{1}\left(S_{R}\right)<\lambda_{1}\left(\Delta_{a, b}^{h}\right)
$$

Proof. We employ a spectral isoperimetric inequality [9, SATZ 3] (see also [10, Theorem 3.9]) to get

$$
\lambda_{1}\left(\Delta_{a, b}^{h}\right) \geq \frac{\pi / 4}{2\left|\Lambda_{a, b}\right|} j_{0}^{2}
$$

where $j_{0} \approx 2.4048$ is the first zero of $J_{0}$, the zeroth Bessel function. The value $\pi / 4$ in the RHS of (5.4) is determined in [10, Theorem 3.9] as the so called 'rotation' of the Neumann boundary of $\Delta_{a, b}^{h}$ (which is the curve $v \cup \gamma_{a, b}$ ). The definition of the rotation of a curve is given in [10, Section 2.3] and in our case, due to the concavity of $\gamma_{a, b}$ it simply equals to the opening angle of $\partial \Lambda_{a, b}$ at $(0, b)$. We note that $\lambda_{1}\left(S_{R}\right)$ is equal to the first Dirichlet eigenvalue of the disc of radius $R$. Namely,

$$
\lambda_{1}\left(S_{R}\right)=\left(\frac{j_{0}}{R}\right)^{2}=j_{0}^{2} \frac{\pi / 8}{\left|\Lambda_{a, b}\right|},
$$

which is the RHS of (5.4), so we get $\lambda_{1}\left(S_{R}\right) \leq \lambda_{1}\left(\Delta_{a, b}^{h}\right)$. This inequality is actually strict since $\Lambda_{a, b}$ is not a circular sector [9, SATZ 3].

Lemma 5.2. There exists $c>1$ such that if $\frac{a}{b}>c$ then

$$
\lambda_{1}\left(\Delta_{a, b}^{v}\right)<\lambda_{1}\left(S_{R}\right) .
$$

Proof. We start by observing that $\lambda_{1}\left(\Delta_{a, b}^{v}\right)=\frac{\pi^{2}}{4}\left(\frac{1}{a^{2}}+\frac{1}{b^{2}}\right)$ (see (1.6)). Indeed, $\frac{\pi^{2}}{4}\left(\frac{1}{a^{2}}+\frac{1}{b^{2}}\right)$ is the eigenvalue corresponding to the eigenfunction $f_{a, b}(x, y)=\sin \left(\frac{\pi}{2 a} x\right) \cos \left(\frac{\pi}{2 b} y\right)$ on $\Omega_{a, b}^{\text {star }}$ (see (1.8)). The restriction $\left.f_{a, b,}\right|_{\Lambda_{a, b}}$ is in the domain of the operator $\Delta_{a, b}^{v}$ since it fulfills Dirichlet boundary conditions at $v$ and Neumann boundary conditions at $h$ (and arguing

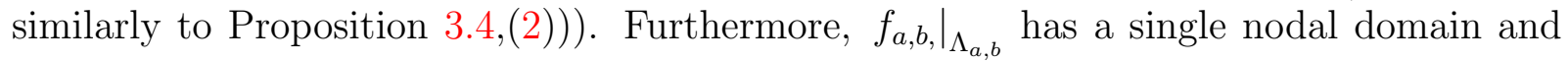
hence it is the first eigenfunction, so that $\lambda_{1}\left(\Delta_{a, b}^{v}\right)=\frac{\pi^{2}}{4}\left(\frac{1}{a^{2}}+\frac{1}{b^{2}}\right)$. 
Noting $\lambda_{1}\left(S_{R}\right)=j_{0}^{2} \frac{\pi / 8}{\left|\Lambda_{a, b}\right|}($ see $(5.5))$, means that (5.6) is equivalent to

$$
\left(\frac{1}{a^{2}}+\frac{1}{b^{2}}\right)\left|\Lambda_{a, b}\right|<\frac{\left(j_{0}\right)^{2}}{2 \pi} .
$$

We defer this last part of the proof to Lemma A.4, where it is shown that there exists $c>1$ such that (5.7) holds whenever $\frac{a}{b}>c$.

Proposition 4.2 now follows as an immediate implication of Lemmata 5.1 and 5.2.

\section{THE AREA-TO-PERIMETER RATIO AND NUMERICS}

In this section we introduce a geometric parameter (a normalized area-to-perimeter ratio) which promotes a further investigation of the spectral position from a numerical perspective.

\subsection{The normalized area-to-perimeter ratio.}

Definition 6.1. [13] Let $f$ be a Morse eigenfunction corresponding to the eigenvalue $\lambda$ and let $\Omega$ be a Neumann domain of $f$. We define the normalized area to perimeter ratio of $\Omega$ by

$$
\rho(\Omega):=\frac{|\Omega|}{|\partial \Omega|} \sqrt{\lambda}
$$

with $|\Omega|$ being the area of $\Omega$ and $|\partial \Omega|$ the total length of its perimeter.

This parameter was originally introduced in [13] for the study of nodal domain geometry. If we consider merely the area to perimeter ratio (without normalizing by the eigenvalue), then the value $\frac{|\Omega|}{|\partial \Omega|}$ has an interesting geometric meaning [25], being equal to the mean chord length of the two-dimensional shape $\Omega$ (up to a multiplicative factor of $\frac{1}{\pi}$ ). The mean chord length is defined as follows; consider all the parallel chords in a chosen direction and take their average length; the uniform average of this value over all directions is the mean chord length ${ }^{3}$.

Interestingly, the value of $\rho(\Omega)$ is also connected to the spectral position of the Neumann domain, $\Omega$.

Proposition 6.2. Let $f$ be a Morse eigenfunction corresponding to eigenvalue $\lambda$. Let $\Omega$ be a Neumann domain of $f$. We have

(1) $\rho(\Omega) \leq \sqrt{2 N_{\Omega}(\lambda)}$.

(2) if $N_{\Omega}(\lambda)=1$ then $\rho(\Omega) \leq \frac{j^{\prime}}{2} \approx 0.9206$

(3) if $N_{\Omega}(\lambda)=2$ then $\rho(\Omega) \leq \frac{j^{\prime}}{\sqrt{2}} \approx 1.3019$,

where $j^{\prime} \approx 1.8412$ is the first zero of the derivative of $J_{1}$, the first Bessel function.

Proof. We write $\rho(\Omega)=\frac{|\Omega|}{|\partial \Omega|} \sqrt{\lambda}=\frac{\sqrt{|\Omega|}}{|\partial \Omega|} \sqrt{\lambda|\Omega|}$. The first factor in this product, is bounded from above by the classical geometric isoperimetric inequality $\frac{\sqrt{|\Omega|}}{|\partial \Omega|} \leq \frac{1}{2 \sqrt{\pi}}[14]$,[22, Theorem 14.1]. An equality occurs if and only is $\Omega$ is a disc. The second factor is bounded from above in terms of the spectral position [19]

$$
\lambda|\Omega| \leq 8 \pi N_{\Omega}(\lambda),
$$

\footnotetext{
${ }^{3}$ We thank John Hannay for pointing out this interesting geometrical meaning to us.
} 
and combining both we get the first bound of the proposition. In the particular cases of $N_{\Omega}(\lambda)=1$ or $N_{\Omega}(\lambda)=2$ the bound (6.1) may be improved as follows.

By the Szegö-Weinberger inequality [34, 36] we have that if $N_{\Omega}(\lambda)=1$ then

$$
\lambda|\Omega| \leq \pi\left(j^{\prime}\right)^{2} .
$$

Combining this bound with the geometric isoperimetric inequality proves the second part of the proposition.

By the Giraurd-Nadirashvili-Polterovich inequality [17] we have that if $N_{\Omega}(\lambda)=2$ then

$$
\lambda|\Omega| \leq 2 \pi\left(j^{\prime}\right)^{2} .
$$

Combining this bound with the geometric isoperimetric inequality proves the third part of the proposition.

The last proposition allows to use numerics to estimate the spectral position of Neumann domains. We note that the exact value of the spectral position cannot be easily computed not even numerically. Part of the difficulty arises since for a general Neumann domain we do not have an analytic expression of its boundary. So, computing the spectrum is highly nontrivial. As apposed to that, the area-to-perimeter ratio is relatively easily computed when the Neumann lines of an eigenfunction are numerically found. Once calculating $\rho(\Omega)$, the last proposition allows to deduce that Neumann domains whose $\rho(\Omega)$ value is large enough do not have low spectral positions.

6.2. Numerical results for separable eigenfunctions. We use the parameter $\rho$ to further investigate the spectral positions of Neumann domains of separable eigenfunctions on the torus. As we have seen in previous sections, a particular separable eigenfunction has only two congruence classes of Neumann domains, the lens-like and the star-like (Figure 1.2). So each separable eigenfunction have just two possible $\rho$ values, $\rho\left(\Omega_{a, b}^{\text {star }}\right)$ and $\rho\left(\Omega_{a, b}^{\text {lens }}\right)$. These $\rho$ values change with the eigenfunction and solely depend on the ratio $a / b$, where $a, b$ are the values which characterize the eigenfunction, (1.8).

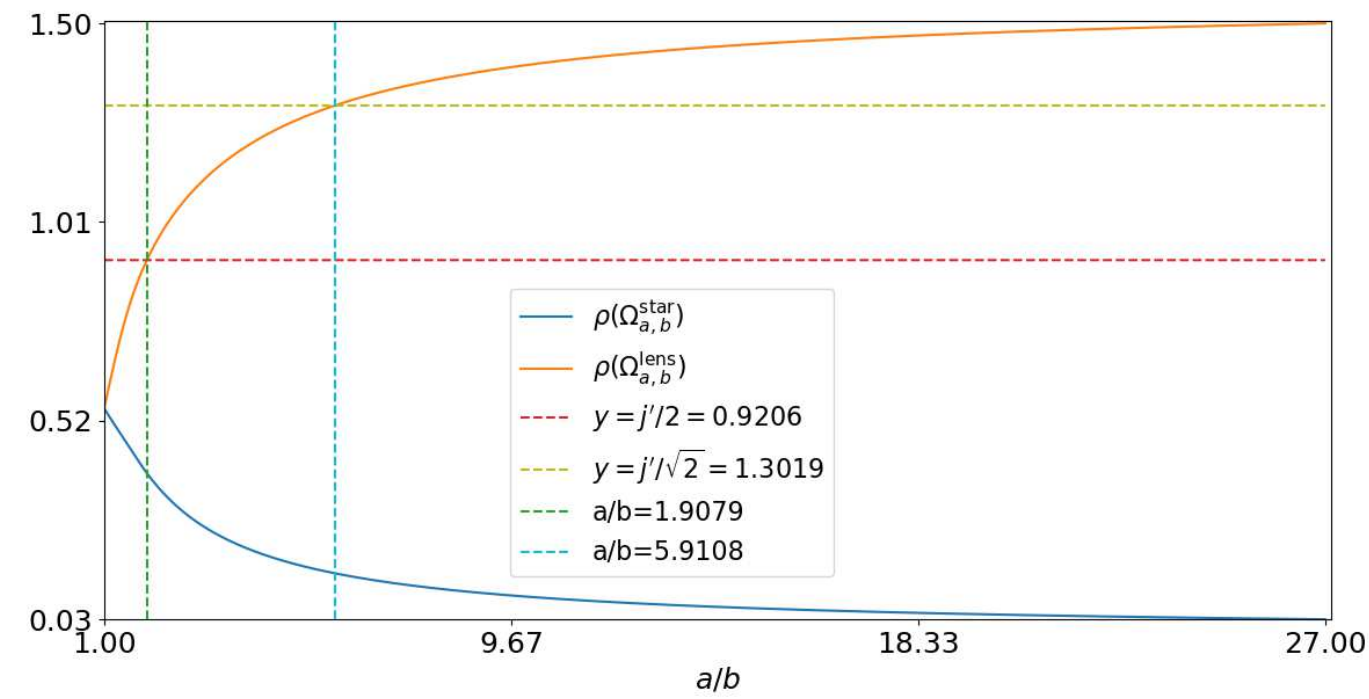

FiguRE 6.1. The values $\rho\left(\Omega_{a, b}^{\text {star }}\right)$ and $\rho\left(\Omega_{a, b}^{\text {lens }}\right)$ for the two Neumann domains of the separable eigenfunction $f_{a, b},(1.8)$, as a function of the ratio $a / b$. The critical $\rho$ values $0.9206,1.3019$ from Proposition 6.2 are marked. 
Figure 6.1 shows how the values $\rho\left(\Omega_{a, b}^{\text {star }}\right)$ and $\rho\left(\Omega_{a, b}^{\text {lens }}\right)$ depend on $a / b$. In particular, we observe that $\rho\left(\Omega_{a, b}^{\text {lens }}\right)$ increases with $a / b$. Using Proposition 6.2 we may conclude that if $a / b>1.9079$ then $N_{\Omega_{a, b}^{\text {lens }}}\left(\lambda_{a, b}\right)>1$. Similarly, if $a / b>5.9108$ then $N_{\Omega_{a, b}^{\text {lens }}}\left(\lambda_{a, b}\right)>2$. Note that according to Theorem 1.4,(1) $N_{\Omega_{a, b}^{\text {lens }}}\left(\lambda_{a, b}\right) \rightarrow \infty$ as $a / b \rightarrow \infty$. The numerical values above allow to slightly refine this result and estimate the growth rate of $N_{\Omega_{a, b}^{\text {lens }}}\left(\lambda_{a, b}\right)$ with $a / b$.

6.3. Numerical results for random waves on the torus. We start by demonstrating an application of Proposition 6.2 to estimate spectral positions of arbitrary Neumann domains. As an example, we show in Figure 6.2 the Neumann partition of an eigenfunction with $\lambda=25$ on the two-dimensional torus. On the left part, only the Neumann partition is shown. On the middle figure each Neumann domain $\Omega$ is colored according to its $\rho(\Omega)$ value and on the right we keep colored only Neumann domains with $\rho(\Omega)>j^{\prime} / 2$ (the rest are left colored blue-red, as in the left figure). From Proposition 6.2,(2) we deduce that all colored Neumann domains have spectral position larger than one.
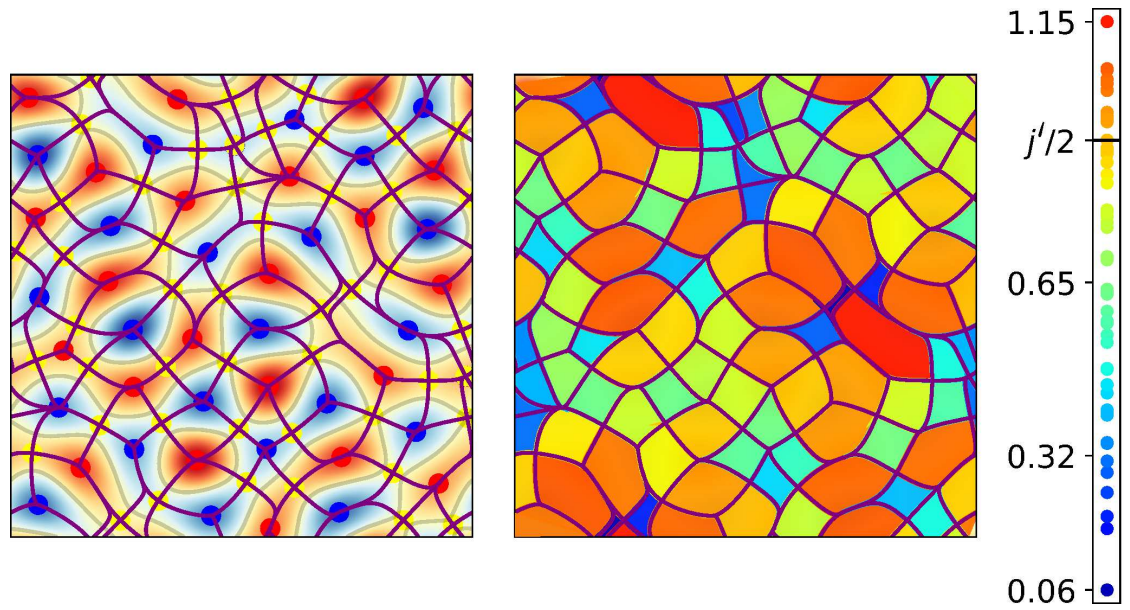

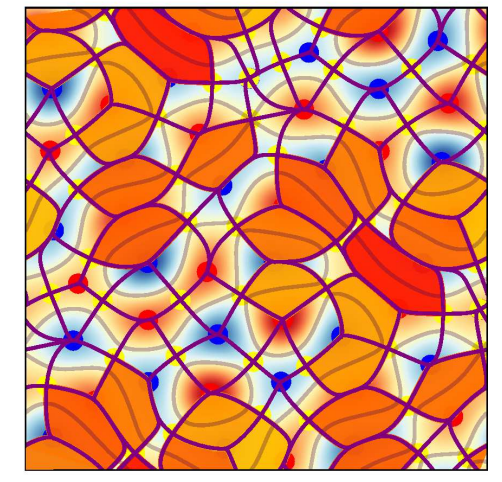

FiguRE 6.2. Left: The Neumann partition of an eigenfunction with $\lambda=25$ on the two-dimensional torus.

Middle: The Neumann domains colored according to their $\rho$ value.

Right: Only Neumann domains for which $\rho(\Omega)>\frac{j^{\prime}}{2}$ are colored according to their $\rho$ value. The rest are colored as in the left part of the figure.

Following this pictorial demonstration, we also calculate the probability distribution of $\rho(\Omega)$ values. We follow the random wave model in our computations. We choose a certain non-simple eigenvalue of the torus. We consider a certain basis of eigenfunctions of this eigenspace and take linear combinations of those eigenfunctions, where the coefficients are chosen according to the standard normal distribution. This describes the random ensemble that we use. We pick approximately 9000 eigenfunctions from this ensemble and for each we calculate $\rho$ values for all of its Neumann domains to obtain the probability distribution of $\rho(\Omega)$ for this particular eigenvalue. 

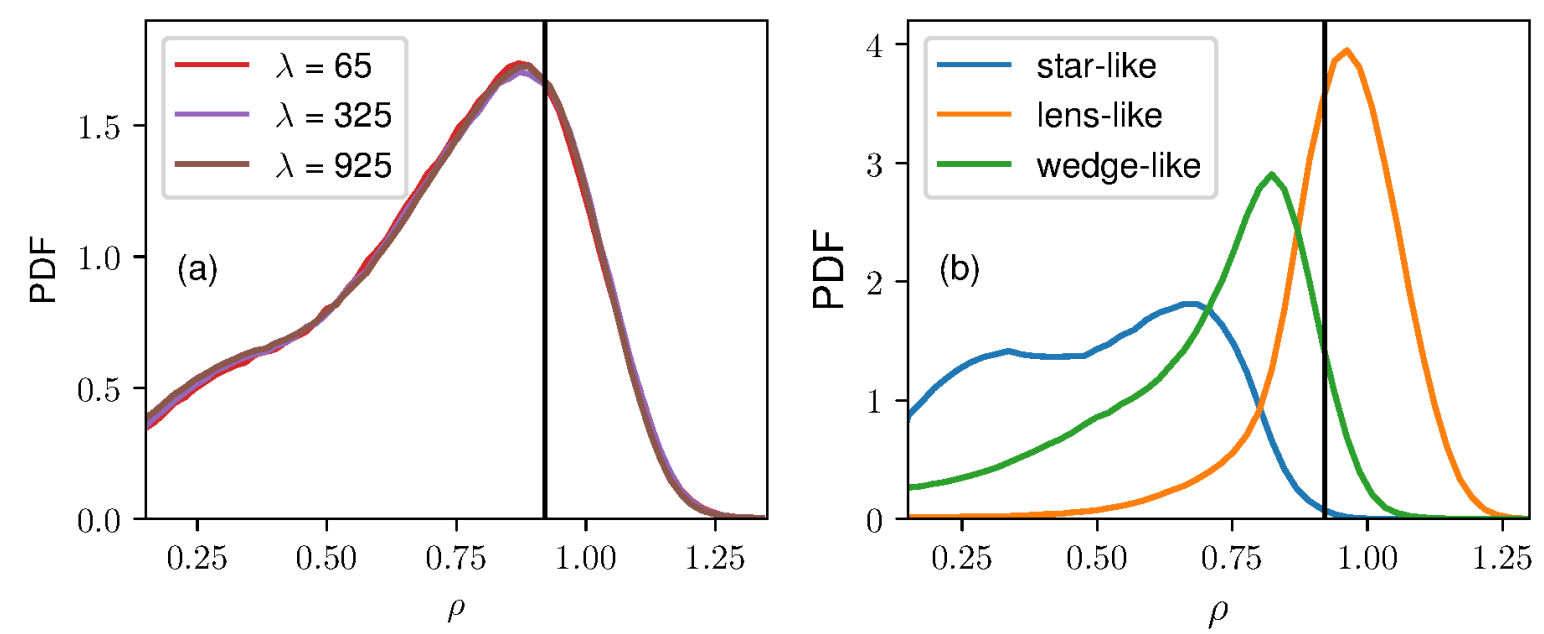

FIguRE 6.3. Left: The probability distribution of $\rho$ values for Neumann domains of random eigenfunctions drawn from three different eigenvalues $(\lambda \in\{65,325,925\})$. The vertical lines marks the bound $j^{\prime} / 2$ from Proposition 6.2,(2).

Right: The probability distributions of $\rho$ values for Neumann domains separated according to the type of the Neumann domain (star, lens, wedge). The random eigenfunctions are of eigenvalue $\lambda=925$.

The results are drawn according to 2494622 lens-like domains, 2670896 starlike domains and 3283304 wedge-like domains, numerically traced and analyzed from approximately 9000 individual eigenfunctions.

The results are shown in Figure 6.3. On the left we plot the probability distribution of three different eigenvalues $(65,325,925)$ of the flat torus of side length $2 \pi$. A first observation is that a substantial proportion (around 23 percent) of the Neumann domains have a $\rho$ value which is larger than $j^{\prime} / 2$ (indicated by a vertical line in the figure), the upper bound in Proposition 6.2,(2). A combination of this numerical observation and the Proposition shows that at least some 23 percent of the Neumann domains have spectral position larger than one. Furthermore, this plots suggests that the $\rho$ distribution might be independent of the particular eigenvalue. We currently do not have an analytic explanation to this numeric finding.

Another interesting observation can be made from the right part of Figure 6.3, which separately shows the $\rho$ distribution of three different types of Neumann domains. Neumann domains may be classified into three types according to the angles their boundary forms at the critical points. Neumann lines always meet perpendicularly at saddle points, whereas at extremal points Neumann lines might meet either at an angle of $\pi$ or of $0,[26,5$, Proposition 4.1]. If both angles at minimum and maximum points of a Neumann domain are 0 we call the Neumann domain star-like. If both angles are $\pi$ we call it lens-like and if one angle is 0 and the other is $\pi$ then the Neumann domain is called wedge-like (see right part of Figure 1.1). With this distinction, we may compute the $\rho$ values for each of the three types above separately (as before, according to the random wave model). Doing so for random eigenfunctions of the eigenvalue 925 results with the right plot in Figure 6.3. This plot might indicate that this three-fold classification affects the geometry of Neumann domains and might suggest a direction for exploring the probability distribution of $\rho$ and its possible universality. 
We end by pointing out the role numerics play in our work. That the spectral position may be higher than one is counter-intuitive (see end of Section 1.2) and is stated in Theorem 1.4,(1). Yet, this result concerns to separable eigenfunctions which are exceptional in some sense. One may wonder whether it is only for this special case that the spectral position differs than one or is it more general than that. The numerics we supply here for random eigenfunctions indicate that generically there is a non-negligible probability that a Neumann domain would have a spectral position larger than one. This calls for further investigations of the Neumann domains spectral positions.

Acknowledgment. We would like to thank Emanuel Milman for stimulating discussions and for pointing out helpful references. We thank Gregory Berkolaiko, Mark Dennis and Michael Levitin for interesting discussions in various stages of this ongoing work. We thank Luc Hillairet and Graham cox for pointing out to us the second proof of Theorem 1.4,(1). Band and Egger were supported by ISF (Grant No. 494/14). Taylor was funded by the Leverhulme Trust Research Programme Grant No. RP2013-K-009.

\section{Appendix A. THE BOUndary of $\Omega_{a, b}^{\text {star }}$ AND ITS AREA}

We consider a separable eigenfunction $f_{a, b}$ on the torus, (1.8), and its star-like Neumann domain, $\Omega_{a, b}^{\text {star }}$. In this appendix we derive the explicit expression for the boundary of $\Omega_{a, b}^{\text {star }}$ (Lemma A.1) and show that it is of class C (Lemma A.3). This boundary characterization is needed to justify the application of some Sobolev space analysis (done in Proposition 3.1). Furthermore, we perform here an asymptotic calculation of the $\Omega_{a, b}^{\text {star }}$ area (Lemma A.4) which is used in the proofs of Theorem 1.4,(1) (first proof) and Lemma 5.2.

Lemma A.1. We have

$$
\Omega_{a, b}^{\mathrm{star}}=\left\{(x, y):|x|<a,|y|<\gamma_{a, b}(x)\right\},
$$

where

$$
\gamma_{a, b}(x):=\frac{2 b}{\pi} \arcsin \left(\left[\cos \left(\frac{\pi}{2 a} x\right)\right]^{\left(\frac{a}{b}\right)^{2}}\right) .
$$

Proof. To prove the Lemma we parameterize the Neumann line which connects the extremal point, $(a, 0)$ to the saddle point, $(0, b)$ (see Figure 1.2,(ii)) and show that it is given by (A.1). The other four Neumann lines which form the boundary of $\Omega_{a, b}^{\text {star }}$ are obtained by noting that $\Omega_{a, b}^{\text {star }}$ is symmetric with respect to horizontal and vertical reflections (see Figure 4.1). Plugging the expression of the eigenfunction (1.8) in the flow equations (1.2) we get

$$
\left(\begin{array}{l}
\dot{x} \\
\dot{y}
\end{array}\right)=-\frac{\pi}{2}\left(\begin{array}{c}
a^{-1} \cos \left(\frac{\pi}{2 a} x\right) \cos \left(\frac{\pi}{2 b} y\right) \\
b^{-1} \sin \left(\frac{\pi}{2 a} x\right) \sin \left(\frac{\pi}{2 b} y\right)
\end{array}\right) .
$$

Hence, the tangent to any gradient flow line is

$$
\frac{\mathrm{d} y}{\mathrm{~d} x}=\frac{a}{b} \tan \left(\frac{\pi}{2 a} x\right) \tan \left(\frac{\pi}{2 b} y\right) .
$$

Integrating this we obtain the gradient flow lines

$$
y(x)=\frac{2 b}{\pi} \arcsin \left(\sin \left(\frac{\pi}{2 b} y_{0}\right)\left[\cos \left(\frac{\pi}{2 a} x\right)\right]^{\left(\frac{a}{b}\right)^{2}}\right),
$$

where $\left(0, y_{0}\right)$ is a point through which the gradient flow line passes. Note that for $-b<y_{0}<b$, each of the gradient flow lines in (A.2) is connected to the extremal point $(a, 0)$, but only 
the one with $y_{0}=b$ is connected to the saddle point $(0, b)$ and hence it is the desired Neumann line ${ }^{4}$.

Remark A.2. From the proof of Lemma A.1 one may also obtain that there is no gradient flow line which connects two saddle points of the eigenfunction $f_{a, b}$. From this we conclude that $f_{a, b}$ is a Morse-Smale function [5, Proposition A.7].

The next lemma shows that the boundary of $\Omega_{a, b}^{\text {star }}$ is regular enough for applying an appropriate Sobolev space analysis. The classification of the boundary in the Lemma is based on [12, Definition 4.1].

Lemma A.3. The boundary of the star-like domain, $\partial \Omega_{a, b}^{\mathrm{star}}$, is of class $C$.

Namely, for any $\boldsymbol{p} \in \partial \Omega_{a, b}^{\text {star }}$ there exists an open neighborhood $U(\boldsymbol{p}) \subset \mathbb{R}^{2}$ and a continuous function $h \in C(I)$ on an interval $I \subset \mathbb{R}$ such that for suitable local Cartesian coordinates

$$
\partial \Omega_{a, b}^{\mathrm{star}} \cap U(\boldsymbol{p})=\{(s, t): t=h(s), s \in I\}
$$

holds.

Proof. Since the boundary consists of gradient flow lines the claim is obvious for every point $\boldsymbol{p}$ not being an end point of such a flow line (i.e, for every $\boldsymbol{p}$ which is not a critical point). At a saddle point any two adjacent Neumann lines meet with an angle of $\frac{\pi}{2}$, [26, Theorem 3.2.]. Hence, the boundary at a neighborhood of a saddle point is also a continuous function. At the extremal points $( \pm a, 0)$ adjacent Neumann lines meet with an angle of 0 and form a cusp. We derive the asymptotics of $\gamma_{a, b}(a-x), x \rightarrow 0^{+}$. Using

$$
\begin{aligned}
\cos \left(\frac{\pi}{2 a}(a-x)\right) & =\sin \left(\frac{\pi}{2 a} x\right)=\frac{\pi}{2 a} x+\mathrm{O}\left(\left(\frac{\pi}{2 a} x\right)^{3}\right) \\
(1+x)^{\beta} & =1+\mathrm{O}(x) \quad \text { for } \beta>0 \\
\arcsin (x) & =x+\mathrm{O}\left(x^{3}\right),
\end{aligned}
$$

we get that for $x \rightarrow 0^{+}$

$$
\text { (A.4) } \gamma_{a, b}(a-x)=\frac{2 b}{\pi} \arcsin \left(\left[\cos \left(\frac{\pi}{2 a}(a-x)\right)\right]^{\left(\frac{a}{b}\right)^{2}}\right)=\frac{2 b}{\pi}\left(\frac{\pi}{2 a} x\right)\left(\frac{a}{b}\right)^{2}+\mathrm{O}\left(x^{3\left(\frac{a}{b}\right)^{2}}\right) \text {. }
$$

These asymptotics show that $\gamma_{a, b}$ is strictly monotonically decreasing in a left neighborhood of $(a, 0)$ and its inverse exists there. Hence, the condition (A.3) is satisfied in a neighborhood of $(a, 0)$ by choosing

$$
h(s)=\left\{\begin{array}{ll}
\gamma_{a, b}^{-1}(s) & s>0 \\
\gamma_{a, b}^{-1}(-s) & s<0
\end{array} .\right.
$$

Finally. we use the expression of $\gamma_{a, b}$ to bound the area of $\Omega_{a, b}^{\text {star }}$ which is needed in the proofs of Theorem 1.4,(1) (first proof) and Lemma 5.2 (see (5.7) in that proof).

Lemma A.4. There exists $c>1$ such if $a / b>c$ then

$$
\frac{1}{a b}\left(\frac{b}{a}+\frac{a}{b}\right)\left|\Omega_{a, b}^{\mathrm{star}}\right|<\frac{2}{\pi}\left(j_{0}\right)^{2}
$$

where $j_{0} \approx 2.4048$ is the first zero of $J_{0}$, the zeroth Bessel function.

\footnotetext{
${ }^{4}$ As a matter of fact, $y_{0}=-b$ also gives a Neumann line, but it is connected to a different saddle point.
} 
Proof. Using Lemma A.1 we have

$$
\begin{aligned}
\frac{1}{a b}\left|\Omega_{a, b}^{\mathrm{star}}\right| & =\frac{8}{\pi a} \int_{0}^{a} \arcsin \left(\left[\cos \left(\frac{\pi}{2 a} x\right)\right]^{\left(\frac{a}{b}\right)^{2}}\right) \mathrm{d} x \\
& =\frac{16}{\pi^{2}} \int_{0}^{\pi / 2} \arcsin \left([\cos (z)]^{\left(\frac{a}{b}\right)^{2}}\right) \mathrm{d} z .
\end{aligned}
$$

We may use the Taylor expansion of $\ln [\cos (z)]$, which converges for $|z|<\frac{\pi}{2}$ (see e.g., $[1$, 4.3.72] and [23, p. 27]) to obtain the bound

$$
\forall z \in\left(0, \frac{\pi}{2}\right), \quad[\cos (z)]^{\left(\frac{a}{b}\right)^{2}}<\exp \left[-\frac{1}{2}\left(\frac{a}{b}\right)^{2} z^{2}\right]
$$

Another bound which we use is

$$
\forall w \in(0,1), \quad \arcsin (w) \leq w+(\pi / 2-1) w^{3} .
$$

To validate (A.8) we may observe that both functions at the RHS and LHS coincide for $w=0$ and $w=1$ and further check that the difference does not vanish anywhere else in $(0,1)$ (for example, since the difference has only a single critical point in this interval).

Plugging the bounds (A.7), (A.8) in (A.6) and using also the monotonicity of $\arcsin (w)$ for $w \in(0,1)$ we get

$$
\begin{aligned}
\frac{1}{a b}\left|\Omega_{a, b}^{\mathrm{star}}\right| & <\frac{16}{\pi^{2}} \int_{0}^{\pi / 2}\left\{\exp \left[-\frac{1}{2}\left(\frac{a}{b}\right)^{2} z^{2}\right]+(\pi / 2-1) \exp \left[-\frac{3}{2}\left(\frac{a}{b}\right)^{2} z^{2}\right]\right\} \mathrm{d} z \\
& <\frac{16}{\pi^{2}} \int_{0}^{\infty}\left\{\exp \left[-\frac{1}{2}\left(\frac{a}{b}\right)^{2} z^{2}\right]+(\pi / 2-1) \exp \left[-\frac{3}{2}\left(\frac{a}{b}\right)^{2} z^{2}\right]\right\} \mathrm{d} z \\
& =\frac{16}{\pi^{2}}\left\{\frac{1}{2} \sqrt{\frac{2 \pi}{(a / b)^{2}}}+(\pi / 2-1) \frac{1}{2} \sqrt{\frac{2 \pi}{3(a / b)^{2}}}\right\} \approx 2.7014 \cdot \frac{b}{a}
\end{aligned}
$$

where moving to the last line we used integration over (half) Gaussian.

From the above we get $\frac{1}{a b}\left(\frac{a}{b}+\frac{b}{a}\right)\left|\Omega_{a, b}^{\text {star }}\right| \lesssim 2.7014 \cdot\left(1+\left(\frac{b}{a}\right)^{2}\right)$. Now, since $\frac{2}{\pi}\left(j_{0}\right)^{2} \approx 3.68$ we get that (A.5) holds if $\frac{b}{a}$ is small enough.

Remark A.5. From the proof one easily gets that (A.5) holds for $a / b \gtrsim\left(\frac{3.68}{2.7}-1\right)^{-1 / 2} \approx 1.66$. Numerically, it seems that choosing $c \approx 1.1407$ already guarantees this bound. This can be seen in Figure A.1. 


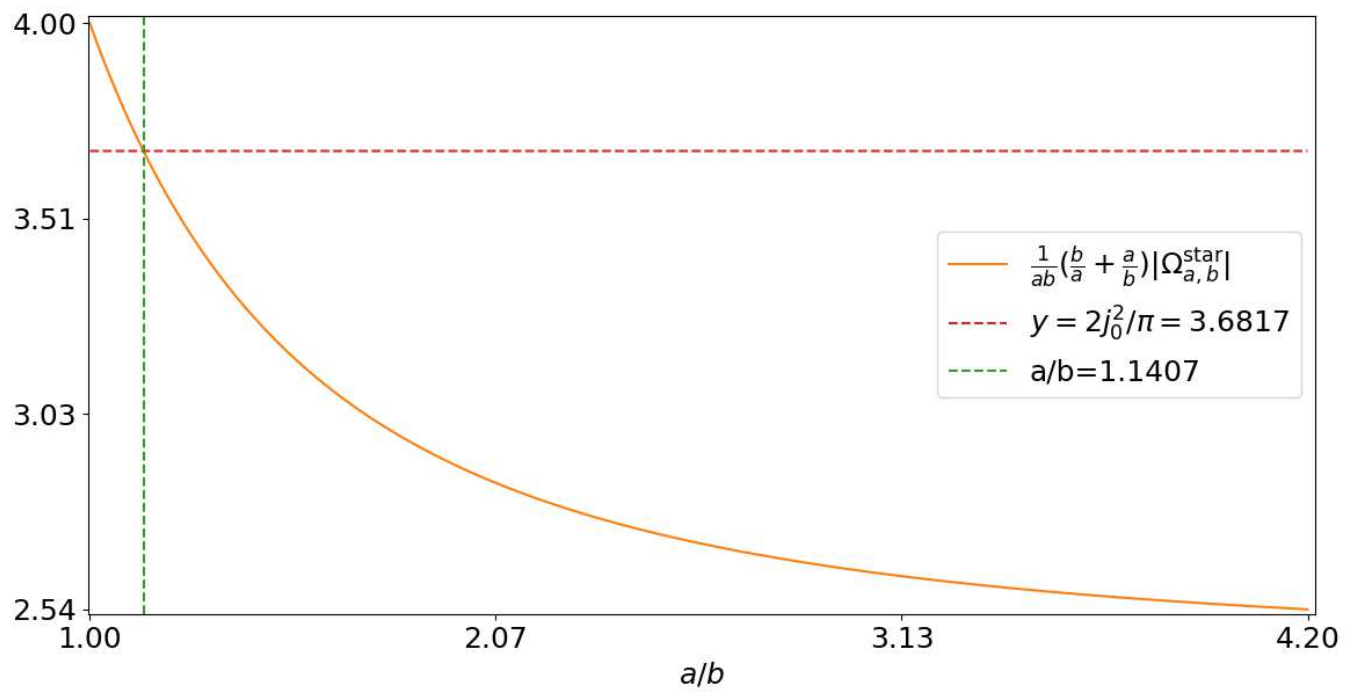

Figure A.1. The left hand side of (A.5) plotted as a function of $a / b$. The right hand side of (A.5) is indicated together with the corresponding $a / b$ value.

\section{REFERENCES}

[1] M. Abramowitz and I. A. Stegun. Handbook of mathematical functions with formulas, graphs, and mathematical tables. For sale by the Superintendent of Documents, U.S. Government Printing Office, Washington, D.C., 1964. 19

[2] G. Acosta, M. G. Armentano, R. G. Durán, and A. L. Lombardi. Nonhomogeneous Neumann problem for the Poisson equation in domains with an external cusp. J. Math. Anal. Appl., 310(2):397-411, 2005. 6

[3] S. Agmon. Lectures on elliptic boundary value problems. AMS Chelsea Publishing, Providence, RI, 2010. Prepared for publication by B. Frank Jones, Jr. with the assistance of George W. Batten, Jr., Revised edition of the 1965 original. 7, 8

[4] J. H. Albert. Topology of the nodal and critical point sets for eigenfunctions of elliptic operators. ProQuest LLC, Ann Arbor, MI, 1972. Thesis (Ph.D.)-Massachusetts Institute of Technology. 1

[5] L. Alon, R. Band, M. Bersudsky, and S. Egger. Neumann Domains on Graphs and Manifolds. arXiv:1805.07612. 3, 16, 18

[6] R. Band, G. Berkolaiko, C. H. Joyner, and W. Liu. Quotients of finite-dimensional operators by symmetry representations. arXiv preprint arXiv:1711.00918, 2017. 9

[7] R. Band, G. Cox, and S. Egger. Spectral properties of Neumann domains via the Dirichlet-toNeumann operator. In preparation. 3

[8] R. Band and D. Fajman. Topological properties of Neumann domains. Ann. Henri Poincaré, 17(9):2379-2407, 2016. 1, 2, 3, 4

[9] C. Bandle. Extremaleigenschaften von Kreissektoren und Halbkugeln. Comment. Math. Helv., 46:356380, 1971. 12

[10] C. Bandle. Isoperimetric inequalities and applications, volume 7 of Monographs and Studies in Mathematics. Pitman (Advanced Publishing Program), Boston, Mass.-London, 1980. 12

[11] R. Courant. Ein allgemeiner Satz zur Theorie der Eigenfunktione selbstadjungierter Differentialausdrücke. Nachr. Ges. Wiss. Göttingen Math Phys, July K1:81-84, 1923. 3, 10, 11

[12] D. E. Edmunds and W. D. Evans. Spectral theory and differential operators. The Clarendon Press, Oxford University Press, New York, 1987. 7, 18

[13] Y. Elon, S. Gnutzmann, C. Joas, and U. Smilansky. Geometric characterization of nodal domains: the area-to-perimeter ratio. J. Phys. A: Math. Theor., 40:2689, 2007. 13

[14] H. Federer. Geometric measure theory. Die Grundlehren der mathematischen Wissenschaften, Band 153. Springer-Verlag New York Inc., New York, 1969. 13 
[15] N. Filonov. On an inequality for the eigenvalues of the Dirichlet and Neumann problems for the Laplace operator. Algebra i Analiz, 16(2):172-176, 2004. 11

[16] L. Friedlander. Some inequalities between Dirichlet and Neumann eigenvalues. Archive for Rational Mechanics and Analysis, 116(2):153-160, 1991. 11

[17] A. Girouard, N. Nadirashvili, and I. Polterovich. Maximization of the second positive Neumann eigenvalue for planar domains. J. Differential Geom., 83(3):637-661, 2009. 14

[18] P. Grisvard. Elliptic problems in nonsmooth domains. SIAM, 2011. 7

[19] P. Kröger. Upper bounds for the Neumann eigenvalues on a bounded domain in Euclidean space. $J$. Funct. Anal., 106(2):353-357, 1992. 5, 13

[20] P. Kuchment and H. Zeng. Convergence of spectra of mesoscopic systems collapsing onto a graph. $J$. Math. Anal. Appl., 258(2):671-700, 2001. 6

[21] P. Kuchment and H. Zeng. Asymptotics of spectra of Neumann Laplacians in thin domains. In Y. Karpeshina, G. Stolz, R. Weikard, and Y. Zeng, editors, Advances in differential equations and mathematical physics (Birmingham, AL, 2002), volume 327 of Contemp. Math., pages 199-213. Amer. Math. Soc., Providence, RI, 2003. 6

[22] F. Maggi. Sets of finite perimeter and geometric variational problems. Cambridge University Press, 2012. 13

[23] W. Magnus, F. Oberhettinger, and R. P. Soni. Formulas and theorems for the special functions of mathematical physics. Springer, 1966. 19

[24] V. G. Maz' ya and S. V. Poborchi. Differentiable functions on bad domains. World Scientific Publishing Co., 1997. 7

[25] A. Mazzolo, C. de Mulatier, and A. Zoia. Cauchy's formulas for random walks in bounded domains. Journal of Mathematical Physics, 55(8):083308, 2014. 13

[26] R. B. McDonald and S. A. Fulling. Neumann nodal domains. Philos. Trans. R. Soc. Lond. Ser. A Math. Phys. Eng. Sci., 372(2007):20120505, 6, 2014. 16, 18

[27] O. Parzanchevski and R. Band. Linear representations and isospectrality with boundary conditions. J. Geom. Anal., 20(2):439-471, 2010. 9

[28] L. E. Payne. Isoperimetric inequalities and their applications. SIAM Rev., 9:453-488, 1967. 11

[29] A. Pleijel. Remarks on Courant's nodal line theorem. Communications on Pure and Applied Mathematics, 9(3):543-550, 1956. 11

[30] G. Pólya. Remarks on the foregoing paper. J. Math. Physics, 31:55-57, 1952. 11

[31] M. Reed and B. Simon. Methods of modern mathematical physics. I-4. Functional analysis. Academic Press, New York, 1972. 6

[32] M. Reed and B. Simon. Methods of modern mathematical physics. Vol. IV. Academic Press, New York, N.Y., 1978. 7

[33] J. Rubinstein and M. Schatzman. Variational problems on multiply connected thin strips. I. Basic estimates and convergence of the Laplacian spectrum. Arch. Ration. Mech. Anal., 160(4):271-308, 2001. 6

[34] G. Szegö. Inequalities for certain eigenvalues of a membrane of given area. J. Rational Mech. Anal., 3:343-356, 1954. 14

[35] K. Uhlenbeck. Generic properties of eigenfunctions. Amer. J. Math., 98(4):1059-1078, 1976. 1

[36] H. F. Weinberger. An isoperimetric inequality for the $N$-dimensional free membrane problem. $J$. Rational Mech. Anal., 5:633-636, 1956. 14

[37] S. Zelditch. Eigenfunctions and nodal sets. Surveys in Differential Geometry, 18:237-308, 2013. 1, 3

${ }^{1}$ Department of Mathematics, Technion-Israel Institute of Technology, Haifa 32000, ISRAEL.

\footnotetext{
${ }^{2}$ This Work Was Carried out While Alexander Taylor Was EMPloyed By the University of Bristol., H H Wills Physics Laboratory, University of Bristol, Tyndall Avenue, Bristol BS8 1TL, United Kingdom.
} 